\title{
Compression of Pedestrian Crowd in Corner Turning Subject experiment-based analysis of walking trajectories
}

\author{
Mineko Imanishi', Tomonori Sano \\ ${ }^{1}$ Fire Protection Engineering Group/Takenaka Research \& Development Institute \\ Otsuka 1-5-1, Inzai, Chiba, Japan \\ imanishi.mineko@takenaka.co.jp \\ Faculty of Human Sciences/Waseda University \\ Mikajima 2-579-1, Tokorozawa, Saitama, Japan \\ sano-t@waseda.jp
}

\begin{abstract}
In this study, pedestrian crowd dynamics at corner turns were investigated by analyzing pedestrian trajectories in a subject experiment for building more reliable, general-purpose, pedestrian simulation models. An experiment under laboratory conditions was conducted wherein a pedestrian crowd walked straight for a short distance before turning into a right-angled corner built with partition walls; the opposite sides were unwalled. Trials were performed with different widths and densities of initial participant positions. Finally, the trajectories of the pedestrians were extracted from a video through computer image analysis. The results demonstrated that pedestrian behavior at corner turns depends on lane position, lane distance (from the wall), and crowd density.
\end{abstract}

Keywords: experiment, crowd dynamics, trajectory, data-set

\section{Introduction}

\subsection{Background}

Pedestrian crowd simulations have mainly been developed in the field of architecture for evacuation safety evaluation and space capacity estimation. For such purposes, flow rates at a bottleneck and congestion in crowded situations play a major role. Meanwhile, the rise of multi-agent simulation over the last decades has led to more visual and realistic simulations in detailed architectural plans. However, visual presentations of pedestrian simulations often depict unnatural-looking agent behaviors in relatively uncrowded situations, which have been made light so far. A typical example of such cases is crowd behavior at a corner. In some evacuation simulation models with potential fields, agents congest too heavily — or even stack - at a corner because they move straight toward an exit, ignoring other possible conditions. These false stacks eventually preclude the correct congestion evaluation of a plan. Crowd behavior at a corner should be modelled based on real pedestrian behavior.

With regard to the pedestrian model at a corner, Chraibi et al. [1] developed a traditional social force model by introducing a "virtual" goal while turning at a corner. They identified a parameter that can control the level of congestion at a corner. However, this mathematical model has yet to be validated using real human behavior. Meanwhile, Dias et al. [2-3] conducted a subject experiment. Their study reported on the walking characteristics (of a single or small pedestrian mass) at a corner by comparing walking speeds and different corner angles. Their results could be strengthened by testing under more natural conditions with a larger pedestrian group to solve the current issue of pedestrian congestion at a corner.

\subsection{Aim}

This study aimed to elucidate pedestrian dynamics at corner turns through a subject experiment for more reliable, general-purpose pedestrian simulation models. 


\section{Methodology}

\subsection{Subject Experiment}

$\mathrm{n}$ this study, the authors conducted an experiment under laboratory conditions in which a pedestrian crowd of 24 participants started walking $7.2 \mathrm{~m}$ before a right-angled corner, turned at the corner naturally, and then continued walking straight for over $4 \mathrm{~m}$. Partition walls of $1.8 \mathrm{~m}$ height were arranged to form the corner, while the adjacent spaces were unwalled (Fig. 1).

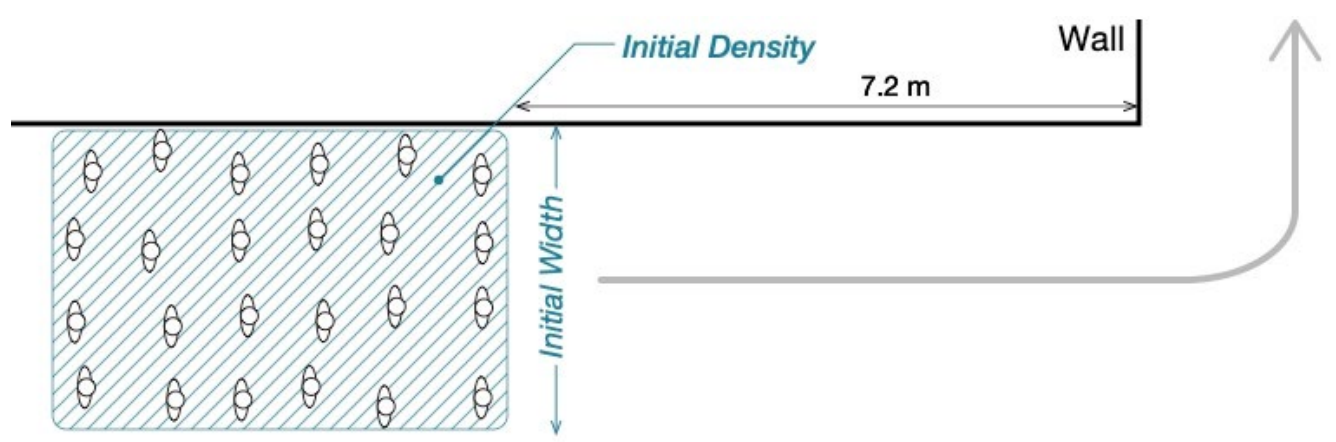

Fig. 1: Schematic of the experiment.

Figure 2 and Table 1 show the experimental conditions. Trials were performed with different widths and densities of initial participant positions. To reproduce a natural situation, the positions were randomized (as shown in Figure 2) by the following process: the participants first lined up along evenly distributed grids marked on the floor with tape; then, they moved one step (or a half-step in high-density conditions) in their desired direction, simultaneously, at a cue from a staff member. Each condition was repeated three times by changing the order of the participants as well as some members. Before the experiment, the participants were instructed: "In these trials, turn left at the corner there (pointing the corner), assuming your destination is beyond the bend. You do not need to maintain formation while walking, and you can overtake others freely. Please behave normally, as though you are walking in a station or shopping mall."

gh

case: Narrow-Middle

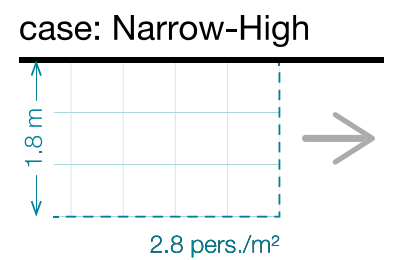

Fig. 2: Initial distribution of participants in each case. 
Table 1: Experimental conditions.

\begin{tabular}{|c|c|c|c|}
\hline Case name & Width & Density & Participants \\
\hline Low & $3.6 \mathrm{~m}$ & 0.7 pers. $/ \mathrm{m}^{2}$ & 24 persons \\
\hline Middle & & 1.2 pers.$/ \mathrm{m}^{2}$ & \\
\hline High & & 2.8 pers. $/ \mathrm{m}^{2}$ & \\
\hline Narrow-Middle & $1.8 \mathrm{~m}$ & 1.2 pers. $/ \mathrm{m}^{2}$ & \\
\hline Narrow-High & & 2.8 pers.$/ \mathrm{m}^{2}$ & \\
\hline
\end{tabular}

Different participants were chosen for each trial from a pool of 60 participants (male: 41 , female: 19). All participants were between 18 and 26 years of age and had no walking disabilities. Fig. 3 shows the population of the participants.

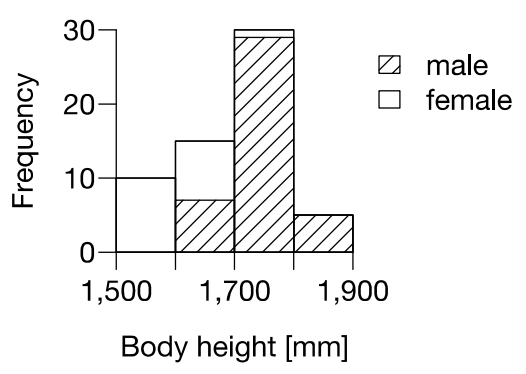

Fig. 3: Distribution of participants according to body height and gender.

The experimental procedure was approved by the Research Ethics Committee at Waseda University, Japan.

\subsection{Data Processing}

The experiment was recorded using a $2.7 \mathrm{~K}$-quality video camera, installed at a height of $15.2 \mathrm{~m}$ from the floor (Figure 4). Colour-tape markers were placed on the head of each participant. Each participant wore one of four possible combinations of tape colors - according to their height - so that the coordinate gaps in still frames due to body height differences could later be corrected using a program. The marker coordinates were obtained by an authors' program [4] that uses OpenCV-an open-source image processing library. Subsequently, the marker locations in the still frames were translated into real-world coordinates by taking the camera lens distortion and participant height differences into account. As such, very accurate coordinates could be obtained at 30 frames per second. 


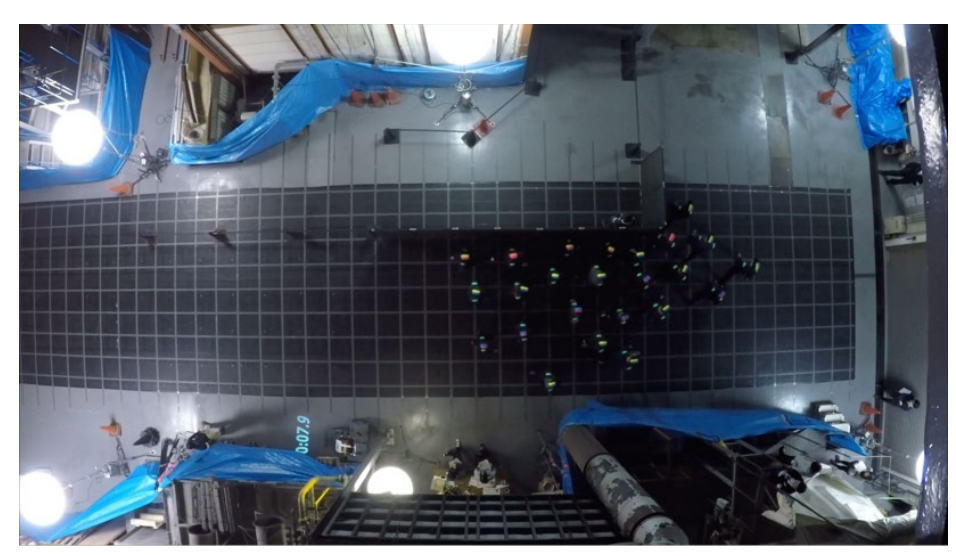

\section{Results}

Fig. 4: A still frame from the experiment video.

\subsection{Compression of pedestrian crowd}

Figures 5-7 show the raw participant trajectories in represented trials of some cases. They illustrate that the pedestrian crowd was compressed once at the corner and then gradually dispersed. The compression ratio of the crowd outline increased as the crowd density decreased. That is, participants preferred to go toward the inside lane (when there was space) to avoid a needless detour. Conversely, participants next to the wall appeared to maintain a regular distance from the wall, regardless of the experimental conditions. Furthermore, those participants momentarily came nearer to the wall when passing the corner. Consequently, the entire pedestrian crowd shifted to the outside of the wall under narrow conditions, whereas the crowd absorbed the inside lane shift under wide conditions.

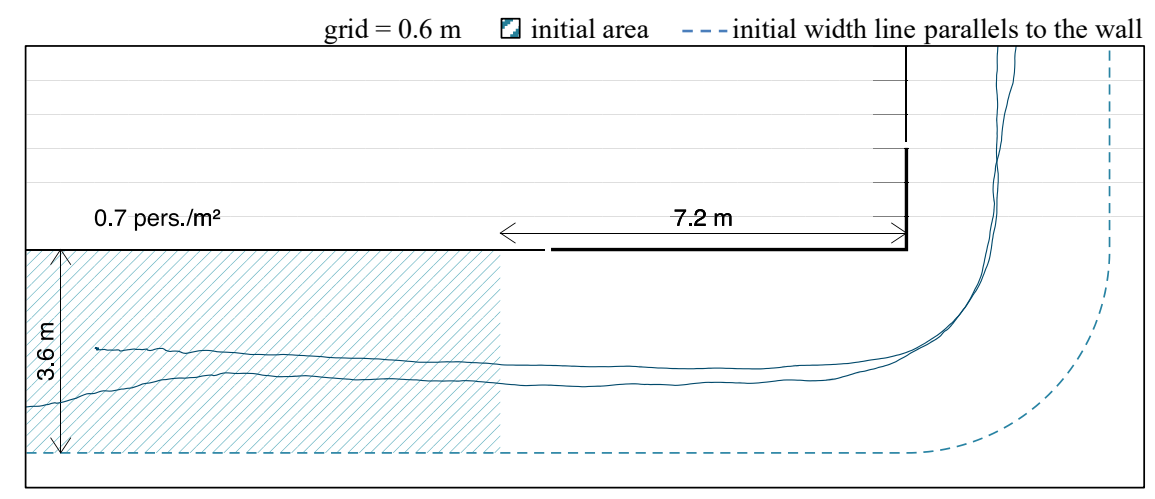

Fig. 5: Walking trajectories of case Low.

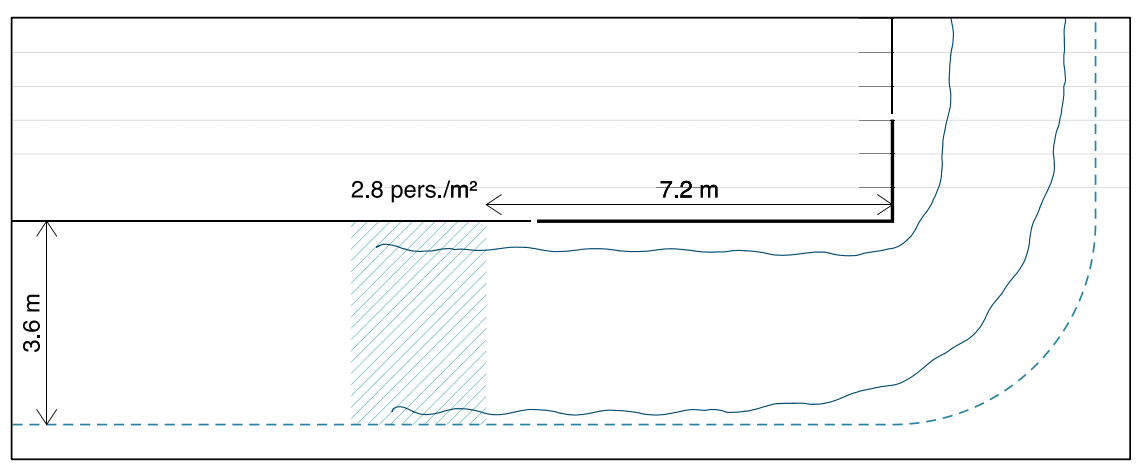


Fig. 6: Walking trajectories of case High.

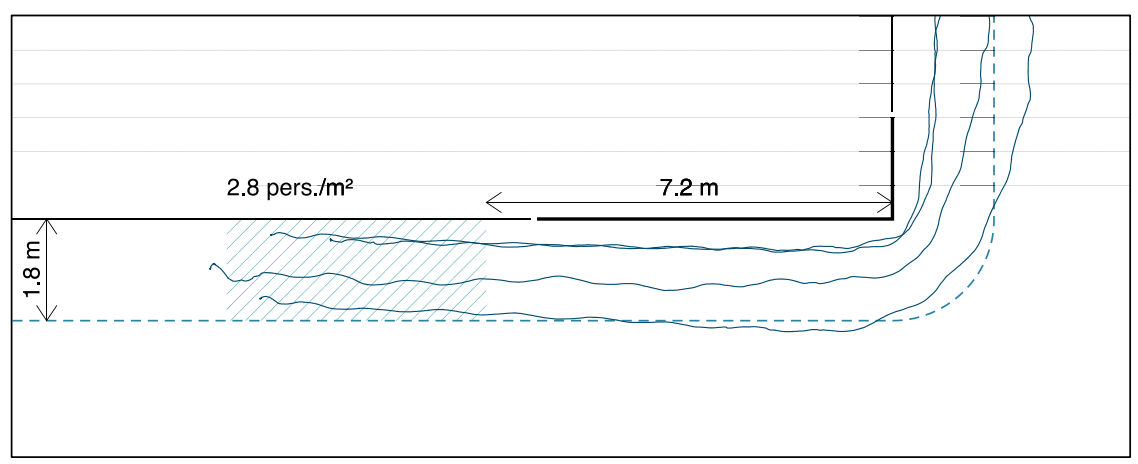

Fig. 7: Walking trajectories of case Narrow-High.

Figure 8 shows each participant's distance from the wall as they passed specific sections defined in the legend plan. Each graph contains all three trials of the respective experiment condition. It indicates that the crowd was compressed - particularly at the middle of the turning phase $\left(45^{\circ}\right.$ section). The pedestrian distribution in the recovery phase (after turning) varied among conditions. It appears that participants did not maintain their original distance from the wall, but rather found a comfortable position by preserving distance from others.
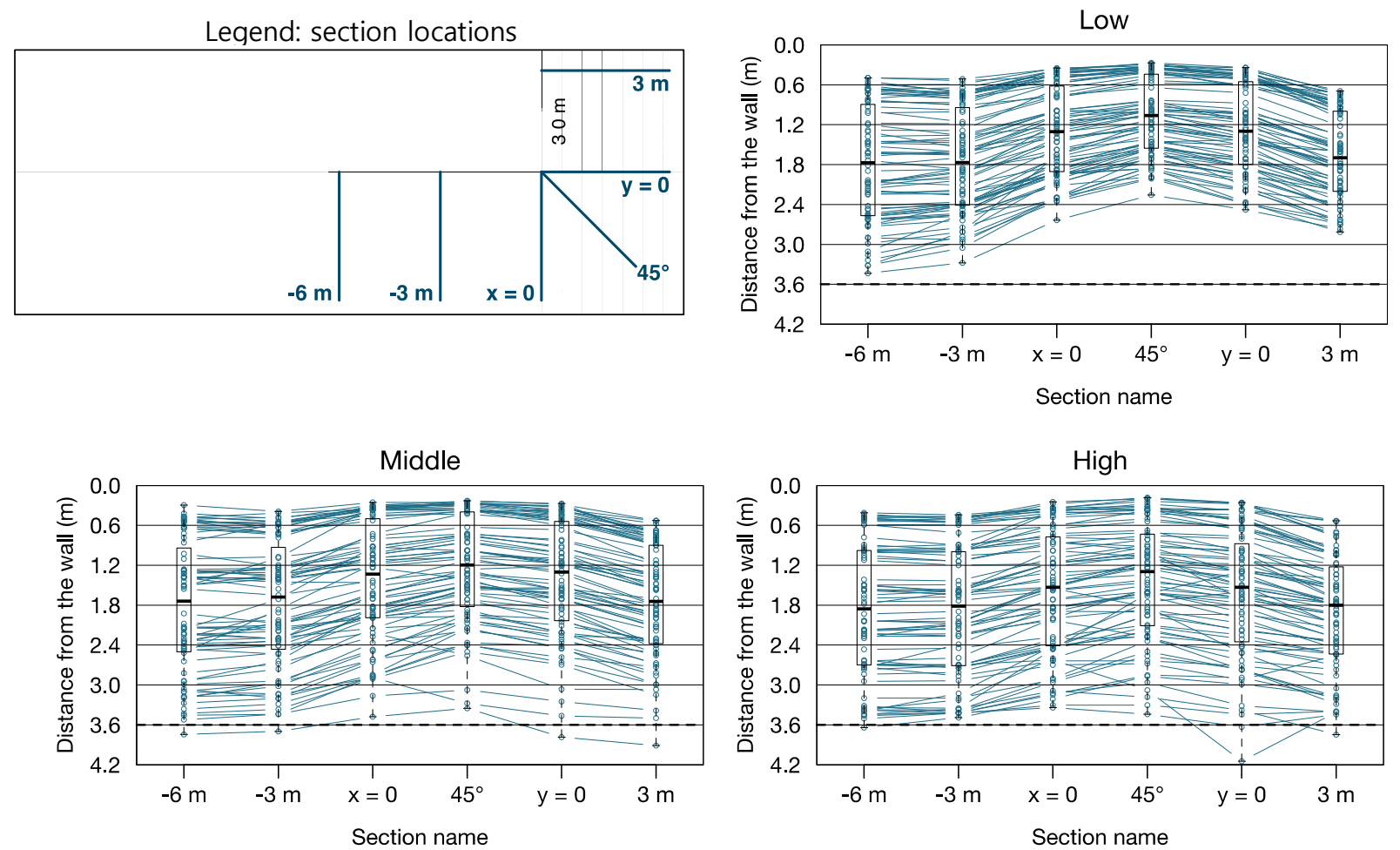

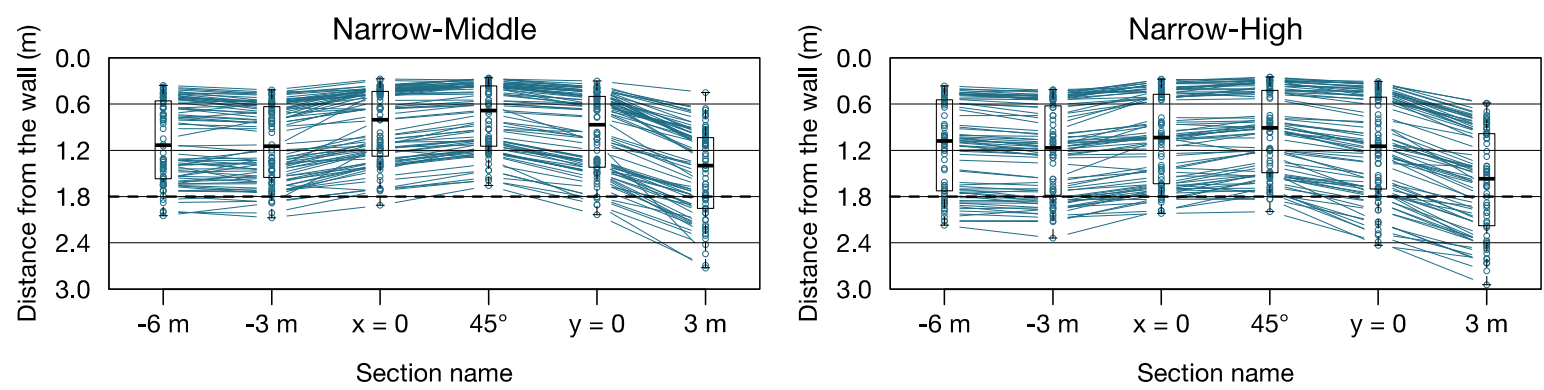

Fig. 8: Distance from the wall at represented sections.

Figure 9 shows the positional relationship between when participants entered $(x=0)$ and exited $(y=0)$ the corner. According to the figure, participants did not change their lane dynamically at the corner. However, the higher the crowd density, the larger the lane variation. This result may suggest that participants were unable to follow their preferred path due to obstacles - other pedestrians. Additionally, in all conditions, the pedestrians concentrated at the edge of the corner because it is the shortest path.
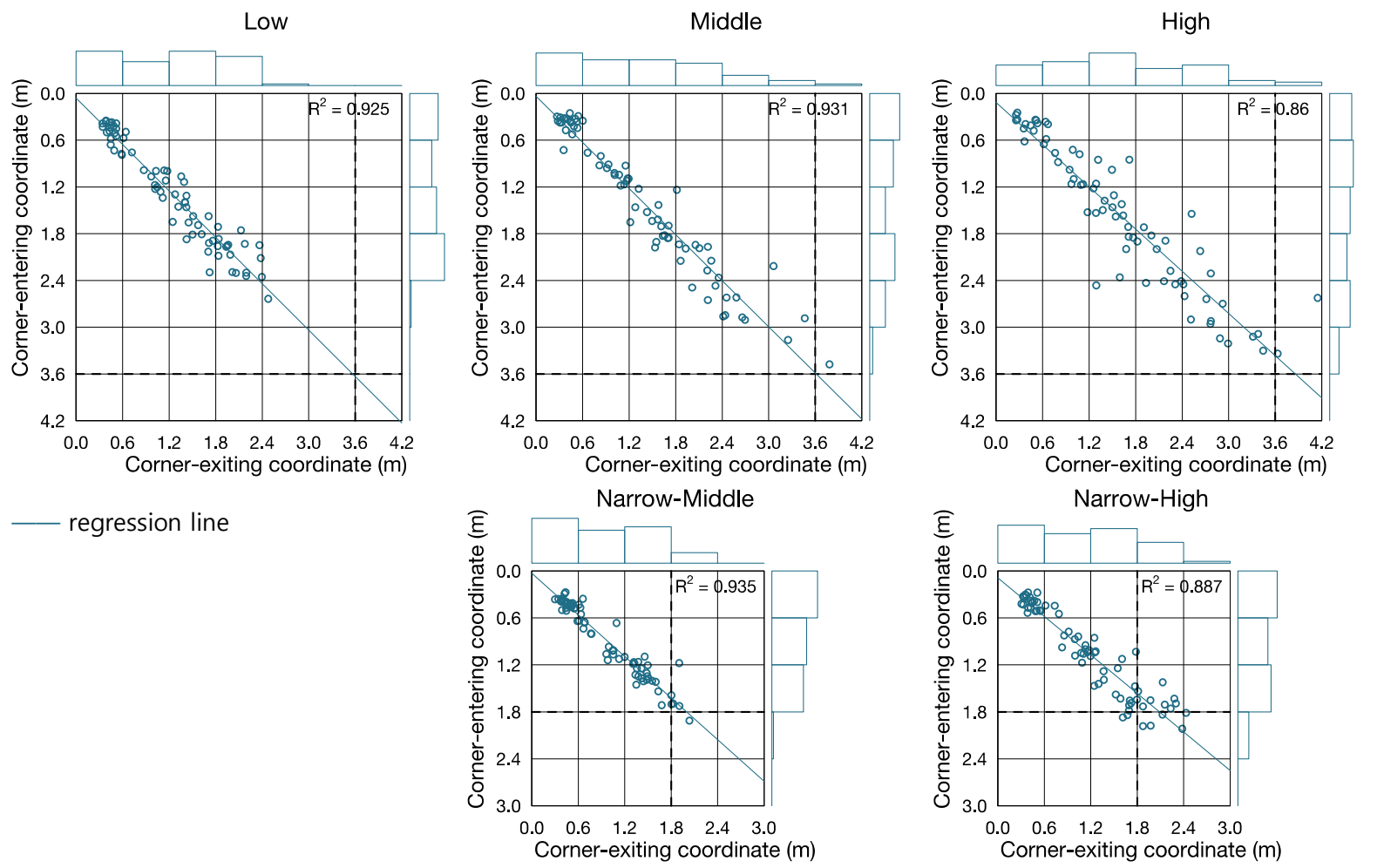

Fig. 9: coordinates perpendicular to the wall when entering and exiting the corner.

\subsection{Pedestrians' walking state differences among location}

Figures 10 and 11 indicate the mean walking directions at each $0.6-\mathrm{m}$ mesh, representative of two complete trials. According to the figure, pedestrians in the outside lane started changing their walking direction earlier than those near the wall. 


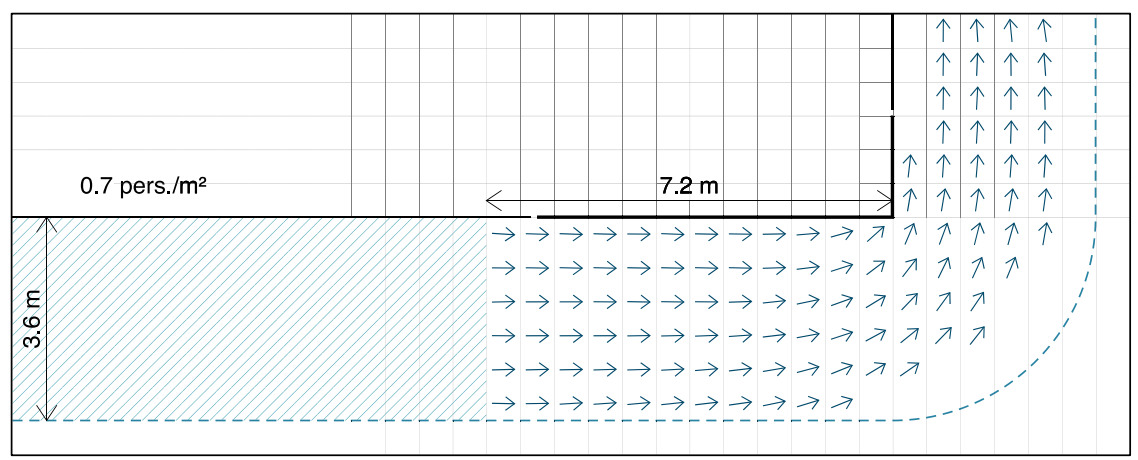

Fig. 10: Distribution of mean walking direction in case Low.

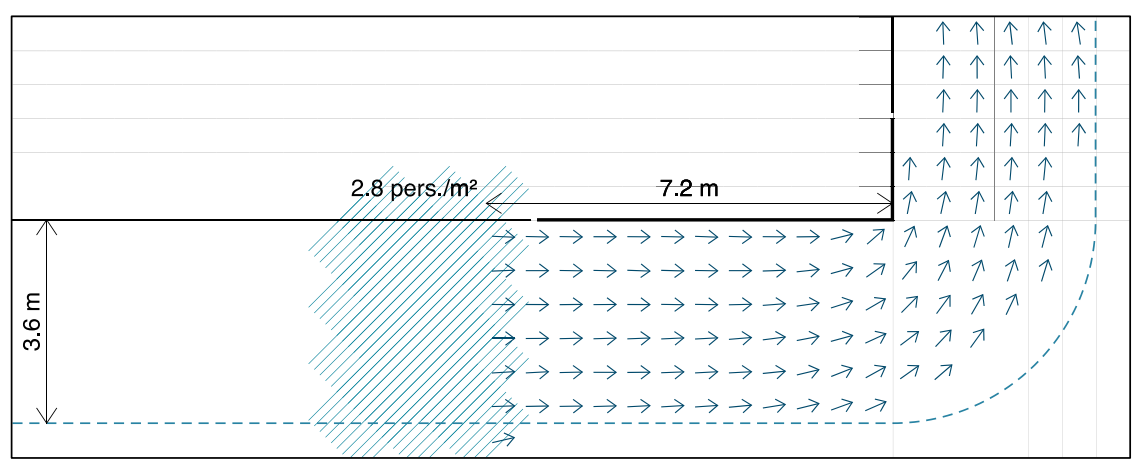

Fig. 11: Distribution of mean walking direction in case High.

Figures 12 and 13 indicate the mean walking speeds of the trials in Figures 10 and 11, respectively. Here, the walking speed of a pedestrian at a location was defined as the moving distance from the location to the location one second later. Participants in the inside lane reduced their speed on turning, while those in the outside lane accelerated. It appears that such variations were induced by a change in crowd density due to the stream direction alternation. Furthermore, the higher the density, the larger the difference between lanes. However, the width reduction was observed to be negligible and far from stacking, even in high-density conditions.

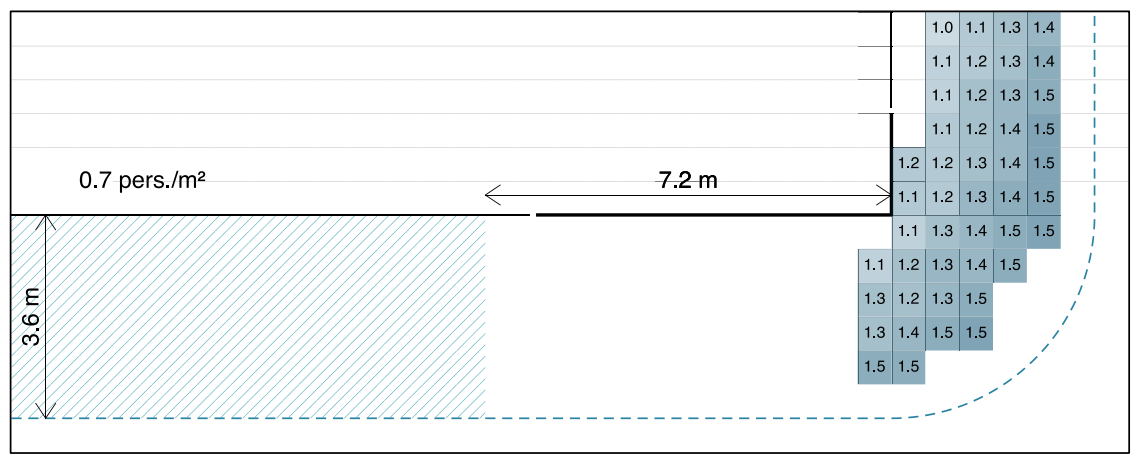

Fig. 12: Distribution of mean walking speed $(\mathrm{m} / \mathrm{s})$ in case Low. 


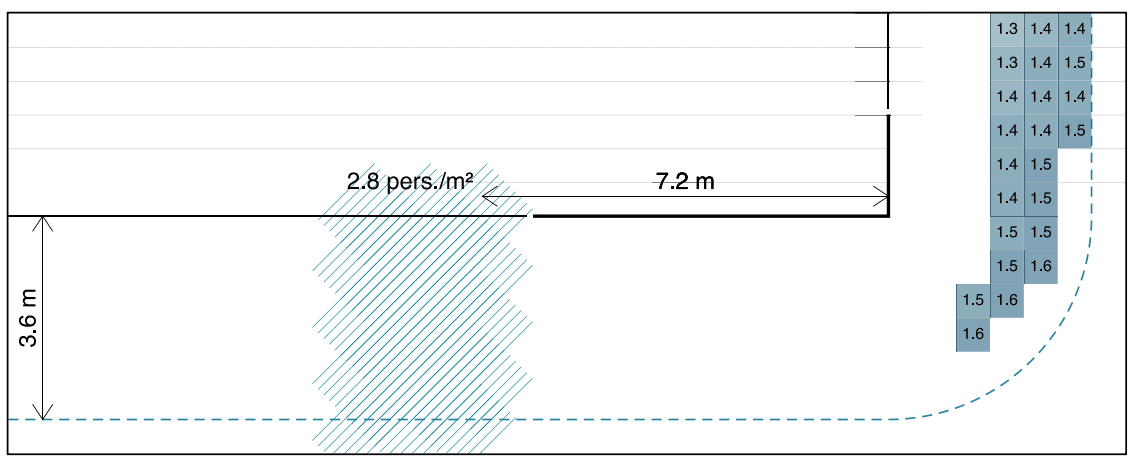

Fig. 13: Distribution of mean walking speed $(\mathrm{m} / \mathrm{s})$ in case High.

\section{Conclusion}

Pedestrian crowd dynamics at corner turns was clarified by analyzing pedestrian trajectories in a subject experiment. The results showed that pedestrian behavior at corner turns depends on lane position, lane distance (from the wall), and crowd density.

Further analysis will be performed on these trajectory data to find a quantitative model that describes crowd behavior at a corner.

\section{Acknowledgements}

This experiment was conducted under a collaborative research agreement between Akeno Facility Resilience Inc., FDM Corporation, Kajima Corporation, National Research Institute of Fire and Disaster, Obayashi Corporation, Shibaura Institute of Technology, Shimizu Corporation, Taisei Corporation, Takenaka Corporation, Tokyo University of Science, and Waseda University (in alphabetical order). This study was supported by the Joint Usage/Research 2017 by the Research Center for Fire Safety Science, which is part of the Organization for Research Advancement of the Tokyo University of Science.

\section{References}

[1] M. Chraibi, M. Freialdenhoven, A. Schadschneider and A. Seyfried, "Modeling the desired direction in a force-based model for pedestrian dynamics," in Traffic and Granular Flow '11, V. Kozlov, A. Buslaev, A. Bugaev, M. Yashina, A. Schadschneider, M. Schreckenberg, Ed. Berlin: Springer, 2013.

[2] C. Dias, M. Sarvi, N. Shiwakoti, and O. Ejtemai, "Experimental Study on Pedestrian Walking Characteristics through Angled Corridors," in Proceedings of 2013 Australasian Transport Research Forum, 2013, pp.1-11.

[3] C. Dias, O. Ejtemai, M. Sarvi, and M. Burd, "Exploring pedestrian walking through angled corridors," Transportation Research Procedia, 2014, vol. 2, pp.19-25.

[4] M. Tange, M. Imanishi, T. Sano, and Y. Ohmiya, "Pedestrian Tracking with Two Different Color Labels for Large Scale Evacuation Experiments," in 12th International Symposium of Fire Safety Science: Book of abstracts posters, Art. P109, June 2017. 